\title{
Urteilsfähigkeit, psychische Störung und Suizid-Beihilfe-Ansinnen
}

\author{
Johann Friedrich Spittler \\ PD Dr. med., ehem. Leitender Oberarzt an der Neurologischen Universitätsklinik der Ruhr-Universität Bochum
}

In der Bevölkerung besteht eine breite Offenheit gegenüber der Sterbehilfe; einzelne Menschen suchen mit gewichtigen Gründen nach Beihilfe zu einem Suizid. Die Ernsthaftigkeit des Ansinnens und die Überzeugungskraft der Argumente müssen ergebnisoffen geprüft und die Fragen einer krankheitswertigen, ggf. therapierbaren psychischen Störung und der Urteilsfähigkeit und Willensfreiheit differenzierend untersucht werden.

\section{Einleitung}

Psychische Störungen sind als weit überwiegende Ursache eines Suizids oder eines Suizidversuchs $[1,2]^{*}$ bekannt. Wenn Menschen bei einer Organisation um Suizid-Beihilfe nachsuchen und aus Gründen der Dokumentation eingehend untersucht werden, kann die Frage einer psychischen Beeinträchtigung der Willensbildung systematisch analysiert werden $[1,3-7]^{* *}$.

Die Literaturangaben finden sich unter www.saez.ch $\rightarrow$ Aktuelle Ausgabe oder $\rightarrow$ Archiv $\rightarrow 2016 \rightarrow 11$.

\footnotetext{
** Der vorliegende Artikel fasst eine Studie zusammen, die als Originalarbeit in der Zeitschrift Nervenheilkunde veröffentlicht wurde [7].
}

\section{Patienten/Methode}

Bei explizitem Suizid-Beihilfe-Ansinnen wurden 494 Menschen zur Frage der "Wohlerwogenheit» $[3,4,6]$ ausführlich ärztlich (und psychiatrisch) untersucht. 75 Personen wurden in Kooperation mit Dignitas, ausschliesslich im Kontext massgeblicher oder begleitender psychischer Störungen, begutachtet. Bei den übrigen Personen, die untersucht worden waren, wurde bezüglich Krankheit oder Begründung keine Selektion

\section{Capacité de discernement, troubles psychiques}

\section{et assistance au suicide}

Objectif de l'étude: Clarifier la question du discernement et de l'autodétermination chez les personnes souffrant de troubles psychiques principaux ou associés qui font appel à une organisation d'assistance au suicide.

Méthodologie: La capacité de discernement, de décision et d'expression de la volonté a été analysée sur la base de 494 expertises psychiatriques.

Résultats: Pour les diagnostics principaux et associés, 206 cas de troubles psychiques de causes et de degrés divers ont été établis. La faculté de discernement a été jugée comme étant maladive dans $8,7 \%$ des cas, psychopathologique, voire sous influence psychodynamique. Tout en étant simultanément rationnelle/réaliste dans $52,4 \%$ des cas, et enfin, équilibrée et rationnelle dans $38,8 \%$ des cas.

vorgenommen. Der psychische Befund wurde mit einer narrativen Schilderung und, angelehnt an das AMDP-Manual mit insgesamt 50 deskriptiven Kriterien, systematisch erfasst. In jedem Fall wurde nach ICD-10 entweder mindestens eine F-Diagnose oder einer der Codes Fyy für eine nicht als krankheitswertig eingeschätzte Trauer oder Fzz für eine unbeeinträchtigte psychische Normalität und Wohlgestimmtheit vergeben. Nicht bei allen Begutachteten wurde eine Beihilfe befürwortet und diese suchten bisher nicht alle endgültig um eine Beihilfe nach [vgl. 9-11].

\section{Resultate}

Die 35,0\% Männer und 65,0\% Frauen standen in einem mittleren Lebensalter von 68 Jahren (Spanne 22-100 Jahre).

Die Begründungen und Motive [7] für die Suizidüberlegungen reichen von körperlichen Leiden über psychische Störungen bis zur Feststellung eines befriedigenden Lebens mit dem Unwillen, sich abzeichnende Einbussen und Abhängigkeiten hinzunehmen (Tab. 1). Die aktuelle Gestimmtheit zur Zeit der gutachtlichen Untersuchung wurde u.a. bezüglich des Antriebs, der depressiv-manischen Dimension, der Angst-Dimension, der Ruhe/Unruhe, der Affektmodulation, der Affektresonanz, des Leidensdrucks, der aktuellen Anspannung, der Affektkontrolle und der Vitalität auf 5- oder 6-stufigen Skalen eingeschätzt [7]. Eine deutlich gehobene oder manische Stimmung wurde nicht beobachtet.

Ein Standardeinwand gegen Suizid-Beihilfe bei psychischen Störungen ist die Forderung nach vorrangiger Therapie. Nach langjähriger Pharmakotherapie einer Psychose oder einer Psychotherapie (bis zu 50 Jahre) 


\begin{tabular}{|c|c|}
\hline $\begin{array}{l}\text { Nur Haupt- } \\
\text { Diagnosen }\end{array}$ & $\begin{array}{l}\text { Mit Neben- } \\
\text { Diagnosen }\end{array}$ \\
\hline $39,1 \%$ & $71,9 \%$ \\
\hline $26,3 \%$ & $41,7 \%$ \\
\hline $1,8 \%$ & $18,4 \%$ \\
\hline
\end{tabular}

bei einer Persönlichkeitsstörung kann eine realistische Erfahrung der Unabänderlichkeit der Verhaltensstörung und der sozialen Desintegration resultieren und in eine meist nur leicht- bis mittelgradige chronische Depression oder auch in eine sehr realistisch einsichtige Trauer münden [7].

Die Urteils- und die selbstbestimmte Willensbildungsfähigkeit wurden nach der Plausibilität und Überzeugungskraft der Selbstdarstellung der Patienten eingeschätzt (Tab. 2). Demnach wurde die Suizid-Beihilfe auch bei psychischer Störung $(n=206)$ in $68,0 \%$ der Fälle als überzeugend zu befürworten, in 15,0\% als zu rechtfertigen und in 7,8\% der Fälle als nicht vertretbar eingeschätzt; in 9,2\% wurde eine Therapie oder eine Wartezeit mit Nachuntersuchung gefordert $[6,13]$.

\section{Diskussion}

\section{Die Eingangsbedingungen}

Von den allgemein beobachteten Suizidversuchs- und Suizidfällen unterscheidet sich das begutachtete Kol- lektiv grundlegend: Die um Beihilfe Nachsuchenden haben sich lange mit Suizid-Gedanken beschäftigt (grundsätzlich erwägend: $16 \pm 16,5$ Jahre, konkret beabsichtigend: $5 \pm 7,3$ Jahre) und verstehen ihr Anliegen als begründet und auch für Dritte überzeugend. Demgemäss handelt es sich weit überwiegend um gefestigte oder subjektiv klar entschiedene Bilanzierungen.

Das vorgestellte Kollektiv ist von den Anforderungsbedingungen bestimmt: 15,2\% der Patienten wurden nach den Vorgaben aus der Schweiz [6] wegen einer (Hauptoder Neben-) Diagnose einer psychischen Störung untersucht, die übrigen Patienten waren bezüglich der zugrunde liegenden Diagnosen unselektiert ${ }^{1}$. Dementsprechend ist der Anteil psychiatrischer Diagnosen relativ erhöht; Schlüsse zu Häufigkeiten innerhalb aller ernsthaften Beihilfe-Ansinnen sind also nur eingeschränkt zulässig.

Alle Untersuchungen und Beurteilungen wurden vom Autor selbst durchgeführt. Im ethischen Konflikt zwischen Lebensschutz und Suizid-Beihilfe liegt die grundsätzliche Präferenz auf Seiten der Selbstbestimmung. Die für die Einschätzung der Urteilsfähigkeit

\footnotetext{
1 In Kooperation mit den

Tabelle 2: Einsichts- und Urteils- und Willensbestimmungsfähigkeit bei Suizid-Beihilfe-Ansinnen im Gesamtkollektiv und bei den psychischen Störungen.

\begin{tabular}{|c|c|c|}
\hline \multicolumn{3}{|l|}{ Die Einsichts- und Urteilsfähigkeit } \\
\hline psychopathologisch, zugleich auch realistisch bestimmt / zu respektieren & $24,7 \%$ & $52,4 \%$ \\
\hline abgewogen rational & $71,7 \%$ & $38,8 \%$ \\
\hline ausschliesslich psychopathologisch & $3,6 \%$ & $7,8 \%$ \\
\hline überwiegend psychopathologisch & $13,6 \%$ & $27,7 \%$ \\
\hline gleichgewichtig psychopathologisch und auch frei-willentlich & $28,9 \%$ & $40,3 \%$ \\
\hline überwiegend frei-willentlich & $25,3 \%$ & $17,0 \%$ \\
\hline ausschliesslich frei-willentlich (autonom) & $28,5 \%$ & $7,3 \%$ \\
\hline
\end{tabular}


für Therapie-Entscheidungen in der Psychiatrie vorgeschlagenen Fragebögen $[8,12]$ können mit kleineren Umformulierungen auf den Suizid mit seiner Endgültigkeit angewendet werden; die Beurteilung der einzelnen Items ist jedoch ebenso dem Ermessen des Beurteilers anheimgestellt, wie die Einschätzung der Urteilsfähigkeit oder der Selbstbestimmtheit insgesamt. Die Instrumente können daher sinnvoll für die Verfeinerung des eigenen Urteilsbildungsprozesses eingesetzt, dürfen aber nicht als schematisch ergebnisbestimmend missverstanden werden.

\section{Gründe und Motive}

Neben konkreten Gründen und Motiven [7] wie dem Leiden an lebenslimitierenden körperlichen Krankheiten (Tab. 1) sind weitere Beweggründe Kinder- und Partnerlosigkeit, Vereinsamung, mangelnder Erfolg somatischer oder psychiatrischer und Psycho-Therapien oder/und die Absehbarkeit von Einschränkungen und insbesondere Abhängigkeit im Alter. Andererseits sind Persönlichkeitsprägungen bedeutsam wie Eigenständigkeit oder Eigenwilligkeit, Duldsamkeit oder Unduldsamkeit, pragmatischer Realismus oder Verdrängungsneigung, Gelassenheit oder Angstbereitschaft und unterschiedliche Radikalität in der Selbstreflexion. Im Einzelfall sind diese Merkmale verschieden ausgeprägt und verstärken oder relativieren sich wechselseitig. Diese Komplexität lässt sich nicht übersichtlich abbilden, muss aber bei der Betrachtung der dargestellten Befunde berücksichtigt werden. Dementsprechend bilden nur die individuellen narrativen Schilderungen mit einer differenzierten kriteriologischen Einordnung, nicht aber die hier nur dargestellten Haupt- und Nebendiagnosen die vielfältigen Bilder angemessen ab.

\section{Therapeutische Möglichkeiten}

Die Mehrzahl der Patienten mit psychischen Störungen war in der Vergangenheit und grossenteils noch aktuell psychopharmakologisch behandelt. Nur ein Teil war von einem Nutzen überzeugt; relativ häufig wurde ein Überwiegen der Nebenwirkungen gegenüber dem Nutzen angegeben. Die grossenteils langjährigen psychiatrischen und Psycho-Therapien wurden teilweise wegen des konsolidierten Vertrauensverhältnisses fortgeführt. Ein Suizid-Beihilfe-Gesuch wurde dem Therapeuten gegenüber vorwiegend aus Unsicherheit wegen dessen unabsehbarer Reaktion $(61,9 \%)$ oder explizit aus Angst vor einer Zwangseinweisung (3,2\%) verheimlicht. Von den 32,4\% ins Vertrauen gezogenen Ärzten waren 2,0\% explizit verständnisvoll (zur Beurkundung eines natürlichen Todes) bereit, keiner jedoch zu einer persönlichen Hilfe. Bei Patienten mit einer psychischen Störung fand aktuell eine psych- iatrische oder Psycho-Therapie statt $(5,8 \%)$, waren die therapeutischen Möglichkeiten ausgeschöpft (17\%), aus psychiatrischer Sicht als realistischerweise nicht aussichtsreich einzuschätzen $(36,4 \%)$ oder bei geringfügiger Störung nicht indiziert (15,5\%); teilweise wurde explizit nur der Suizid angestrebt (6,8\%).

\section{Psychische Störung und selbstbestimmte Willensbildung}

Auch bei bestehender psychischer Störung bzw. Depression war die Einsichts-, Urteils- und Willensfähigkeit deutlich überwiegend hinreichend klar (Tab. 2) $[2,7]$. Die Entscheidung für eine Ablehnung des Gesuchs oder auch für eine Therapie-Auflage erwies sich als problematisch. Bei 2 floriden Psychosen mit eingeschränkter Willensfreiheit kam es zu einsam eigentätigen Suiziden, im einen Fall während der Beurlaubung aus einer psychiatrischen Klinik, im anderen Fall während der Überlegung einer Zwangseinweisung. Nach verzögerter Zusage oder Absage einer Beihilfe mussten weitere 5 spätere einsame Suizide zur Kenntnis genommen werden. Andererseits lebt eine ganze Reihe von ihnen nach Erhalt und in der Sicherheit des "Grünen Lichts» noch weiter, teilweise unter nicht leichten Bedingungen.

\section{Schlussfolgerungen}

Bei einem an eine Organisation herangetragenen Ansinnen auf Suizid-Beihilfe zeigt die eingehende Untersuchung eine breite Variabilität somatischer, psychischer und psychopathologischer Aspekte. Eine pauschale Unterstellung einer aufgrund einer psychischen Störung aufgehobenen oder massgeblich eingeschränkten Urteils- und Willensbildungsfähigkeit ignoriert diese Realität. Jede Situation muss individuell differenzierend beurteilt werden. Die Subjektivität grenzwertiger Beurteilungen wird als grundsätzlich unausweichlich gesehen, muss aber immer auch selbstkritisch abgewogen werden.

Die durch psychische Störungen mögliche Einschränkung der Willensbildung erfordert eine Fürsorge für entsprechend erkrankte und eingeschränkt urteilsfähige Menschen. Wenn ein fürsorglich wohlwollender ärztlicher oder gesellschaftlicher Paternalismus in eine selbstschützende oder abwehrende Fremdbestimmung umschlägt, kann der Patient sich dieser nur mit zielstrebigem Verheimlichen seiner Planungen entziehen. Hier ergeben sich ethisch schwierige Entscheidungen zwischen psychiatrisch-therapeutischer Fürsorge, Schweigepflicht und Selbstbestimmungsrespekt, Entscheidungen mit einer schicksalhaften Dimension.

Disclosure statement

Der Autor gibt an, dass kein Interessenkonflikt besteht. 


\section{Literatur}

1. Arsenault-Lapierre G, Kim C, Turecki G. Psychiatric diagnoses in 3275 suicides: a meta-analysis. BMC Psychiatry 2004;4:37:doi:10.1186/1471-244X-4-37

2. Wolfersdorf M. Suizidalität. Nervenarzt 2008;79:1319-36

3. Bosshard G, Ulrich E, Bär W. 748 cases of suicide assisted by a Swiss right-to-die organisation. Swiss Med Wkly 2003;133:310-7

4. Fischer S, Huber CA, Furter M, Imhof L, Mahrer-Imhof R, Schwarzenegger C et al. Reasons why people in Switzerland seek assisted suicide: the view of patients and physicians. Swiss Med Wkly 2009;139:333-8

5. Lehmann K. Zur Psychologie des Suizids am Beispiel der affektiven und schizophrenen Psychosen. In: Pohlmeier H, Schöch H, Venzlaff U. Hrsg. Suizid zwischen Medizin und Recht. Stuttgart: G. Fischer;1996. S. 135-45

6. Rippe KP, Schwarzenegger C, Bosshard G, Kiesewetter M Urteilsfähigkeit bei Menschen mit psychischen Störungen und Suizidbeihilfe. Schweizer Juristen-Zeitung 2005;101:53-91

7. Spittler JF. Selbstbestimmung und psychische Störung bei Suizid-Beihilfe-Ansinnen. Nervenheilkunde 2015;34:1026-31
8. Vollmann J. Patientenselbstbestimmung und Selbstbestimmungsfähigkeit. Stuttgart, Kohlhammer; 2008

9. Frei A, Schenker TA, Finzen A, Hoffmann-Richter U. Beihilfe zum Suizid bei psychisch Kranken. Nervenarzt 1999;70:1014-1018

10. Lauter H. Probleme und Meinungsstand aus ärztlicher Sicht. In Ritzel G, Beihilfe zum Suizid - Ein Weg im Streit um Sterbehilfe? Regensburg, Roderer; 1998. S. 36-44

11. Strnad J, Grosjean S, Schuepbach B, Bahro M. Suizide in der stationären Psychiatrie unter Beihilfe einer Sterbehilfevereinigung - Fälle aktiver Sterbehilfe? Nervenarzt 1999;70:645-9

12. Wasserfallen J-B, Stiefel F, Clarke S, Crespo A. Appréciation de la capacité de discernement des patients: procédure d'aide à l'usage des médecins. Schweiz Ärztezeitung 2004;85:1701-4

13. Pohlmeier H. Freiverantwortlichkeit des Suizids aus medizinischpsychologischer Sicht. In: Pohlmeier H, Schöch H, Venzlaff U (eds.) Suizid zwischen Medizin und Recht. Stuttgart: G. Fischer, 1996. p. 33-51. 